\title{
Reconstrucción biliogastrointestinal con duodenectomía y preservación de páncreas por laparoscopia posterior a una coledocoduodenostomía por laparotomía fallida
}

\author{
Biliogastrointestinal reconstruction with duodenectomy and pancreas preservation by \\ laparoscopy after open failed coledocoduodenostomy
}

\begin{abstract}
Daniel Gómez ${ }^{1}$ Andrés Mendoza-Zuchini ${ }^{2}$, Mauricio Pedraza ${ }^{2 *}$, Luis F. Cabrera ${ }^{2,3}$ y Jean Pulido-Segura ${ }^{4}$ ${ }^{1}$ Departamento de Cirugía Laparoscópica Avanzada, Universidad Militar Nueva Granada; ${ }^{2}$ Departamento de Cirugía General, Universidad El Bosque; ${ }^{3}$ Departamento Cirugía General, Jose Feliz Patiño, Fundación Santa Fe de Bogotá, Universidad Los Andes; ${ }^{4}$ Departamento de Medicina, Universidad El Bosque. Bogotá, Colombia
\end{abstract}

\section{Resumen}

Las lesiones de la vía biliar y las reconstrucciones biliodigestivas son un reto quirúrgico para el cirujano, además de una situación que exige su máxima habilidad y conocimiento. Presentamos el caso de un paciente con una reconstrucción biliodigestiva por una lesión de vía biliar abierta la cual fue fallida. Se decide llevar a reconstrucción biliodigestiva por laparoscopia, con preservación del páncreas, en un asa con hepatoyeyunostomía y gastroyeyunostomía. Este caso ilustra la posibilidad del manejo con cirugía mínimamente invasiva incluso en los casos más graves; sin embargo, se requiere alta experticia al momento de abordarlo.

Palabras clave: Cirugía. Laparoscopia. Reconstrucción biliodigestiva. Vía biliar común.

\begin{abstract}
Bile duct injury and bile duct reconstruction are a surgical challenge for the surgeon, in addition to a situation that demands maximum skill and knowledge. We present a case of a patient with a biliodigestive reconstruction due to an open bile duct injury which was failed, it was decided to take a biliodigestive reconstruction by laparoscopy, with preservation of the pancreas, with hepaticoyejunostomy and gastroyejunostomy. This case illustrates the possibility of handling with minimally invasive surgery even in the most severe cases, however, they require high expertise when addressing it.
\end{abstract}

Key words: Surgery. Laparoscopy. Biliodigestive reconstruction. Common bile duct.

\section{Introducción}

Desde que la colecistectomía por laparoscopia apareció en la década de 1980 se convirtió en el procedimiento estándar para el manejo de la patología de la vesícula biliar benigna, debido a que su comparación con la técnica abierta tiene mejores resultados en cuanto a dolor posoperatorio, retorno a la actividad del paciente, disminución de las infecciones de sitio operatorio, menor estancia hospitalaria, menor costo y mejor satisfacción del paciente ${ }^{1-3}$. Actualmente se considera uno de los procedimientos más comúnmente realizados en el mundo ${ }^{4}$,

\section{Correspondencia:}

*Mauricio Pedraza

Carrera $72,181-55$

C.P. 111.166, Bogota DC, Colombia 


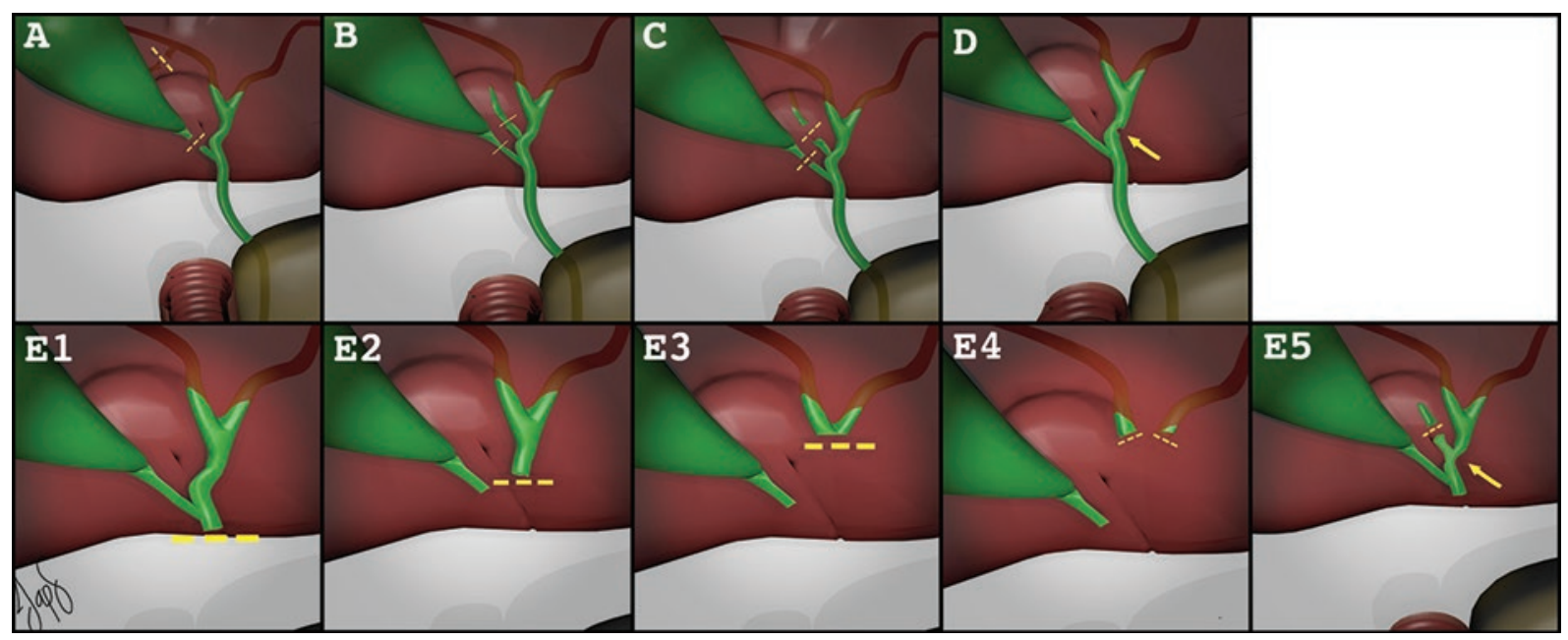

Figura 1. Clasificación de Strasberg para lesiones iatrogénicas de la vía biliar, modelo 3D por JAPS.

con una tasa general de complicaciones que puede oscilar entre un $0,9 \%$ y un $5 \%$ según la serie ${ }^{5}$. La incidencia de lesión de vía biliar con esta técnica va desde el $0.06 \%$ hasta el $1 \%$. 7 . Desde la década de 1990 se ha estudiado ampliamente cómo puede ocurrir la mayor complicación relacionada con esta cirugía, en especial por Strasberg, et al. ${ }^{6}$, que en 1995 insertaron el método de referencia para la prevención de la lesión de la vía biliar durante la colecistectomía por laparoscopia, llamado «vista crítica de seguridad», que tiene como objetivo la identificación del colédoco y prevenir su lesión. A pesar de los desarrollos en tecnologías y de la mencionada vista crítica de seguridad, en el mundo se siguen presentando lesiones de vía biliar. En los Estados Unidos se realizan aproximadamente 1,000,000 de colecistectomías por laparoscopia anualmente, lo cual representa entre 2,500 y 3,000 lesiones de vía biliar por año ${ }^{3}$. La lesión de la vía biliar y sus complicaciones, como infecciones, fistula biliar u obstrucción, son devastadoras para el paciente, con una alta morbilidad y una mortalidad a los 3 meses que puede alcanzar el 2,4\% según lo reportado por Pitt, et al. ${ }^{8}$, o presentar una tasa de supervivencia media de 17.6 años y hasta un $20.8 \%$ de mortalidad por cualquier causa en los primeros 30 días $^{9}$. Existen varios tipos de lesiones en la vía biliar y se han creado varias clasificaciones para ellas, pero una de las más usadas es la clasificación de Strasberg (Fig. 1).

Si ya constituye un reto para el cirujano actual prevenir la lesión de la vía biliar, lo es aún más la solución del problema. Aunque la reparación de la vía biliar puede ser satisfactoria, se necesita experiencia en el manejo de estas lesiones. La mayoría de los estudios han demostrado que en manos de un cirujano experto y con reparación en el mismo tiempo quirúrgico de la lesión los resultados serán favorables, dependiendo también del tipo de lesión y si se asocia o no a una lesión vascular. A continuación, se reporta el caso de un paciente a quien se le realizó una reconstrucción biliodigestiva por laparoscopia posterior a una coledocoduodenostomía por laparotomía fallida en un intento de reparación de una lesión de vía biliar E4 de la clasificación de Strasberg, y se hace una revisión de la literatura.

\section{Caso clínico}

Se trata de una paciente de 57 años, sin antecedentes de importancia, llevada a colecistectomía por laparoscopia de urgencias por colecistitis aguda grave de grado III según la última clasificación de las guías de Tokio $2018^{10}$, con hallazgos intraoperatorios de plastrón vesicular con adherencias al colon transverso, el duodeno y el epiplón mayor y piocolecisto. Una vez completada la colecistectomía, se identifica la lesión de la vía biliar a nivel de la confluencia de los hepáticos con extensión al conducto hepático derecho, además de lesión colónica. Se convierte a laparotomía y se realiza anastomosis colocolónica laterolateral con sutura mecánica, así como anastomosis de la vía biliar al duodeno en diamante. La evolución posoperatoria de la paciente no resulta favorable, con fuga biliar con drenajes de aproximadamente $500-600 \mathrm{~cm}^{3}$ cada 24 horas, sin signos de sepsis ni choque séptico, por lo cual se decide la reexploración laparoscópica.

Es reintervenida a los 8 días de posoperatorio y se identifica dehiscencia de más del $50 \%$ de la anastomosis bilioentérica, bilioperitoneo residual y peritonitis 


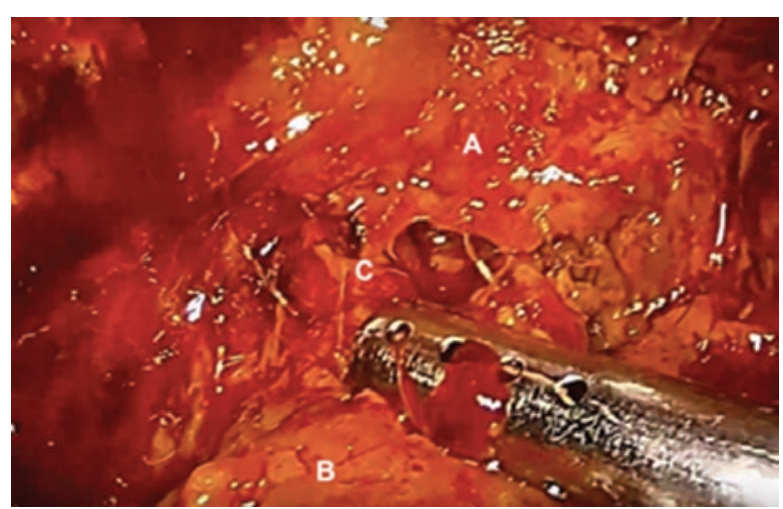

Figura 2. A: Dehiscencia de anastomosis hepatoduodenal. B: placa hiliar duodeno. C: anastomosis dehiscente en la totalidad de su cara anterior.

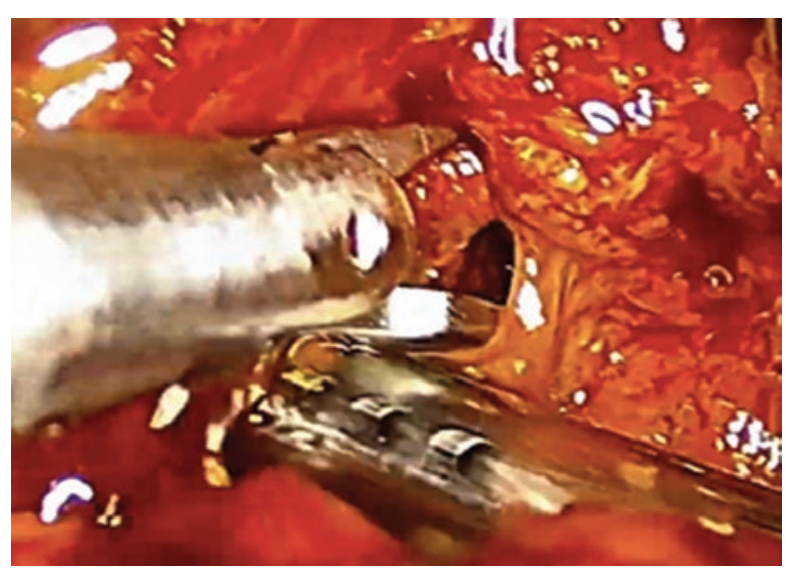

Figura 3. Desmonte de la anastomosis e identificación de la vía biliar.

química localizada por fuga de bilis y contenido duodenal, con importante compromiso inflamatorio y tejidos friables (Fig. 2).

Una vez liberada la anastomosis bilioentérica fallida, se identifican ambos conductos hepáticos y las lesiones previamente descritas (Fig. 3).

Se decide realizar la reconstrucción biliodigestiva por laparoscopia con hepatoyeyunostomía a la placa hiliar (Fig. 4). Después se procede a realizar duodenectomía con preservación del páncreas inmediatamente superior a la vía biliar de la primera y parte de la segunda porción (Fig. 5), y anastomosis gastroyeyunal en la misma asa a $60 \mathrm{~cm}$ de la anastomosis bilioentérica para prevenir el reflujo biliar (Fig. 6).

El tiempo quirúrgico fue de 200 minutos y el sangrado intraoperatorio fue de $50 \mathrm{~cm}^{3}$; no se documentaron lesiones vasculares asociadas ni fuga de la anastomosis colocolónica previa. La evolución posoperatoria fue adecuada, con tolerancia a la vía oral el

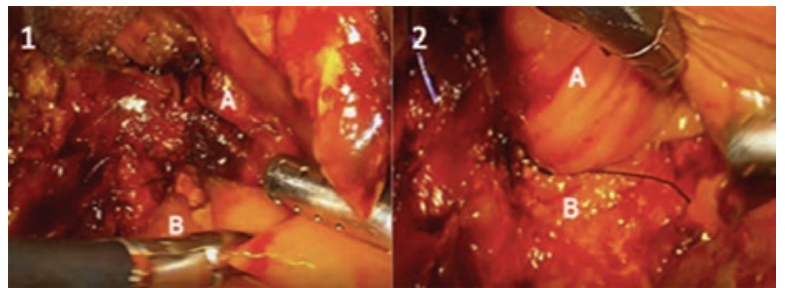

Figura 4. A: Anastomosis hepatoyeyunal a la placa hiliar. B: yeyuno.

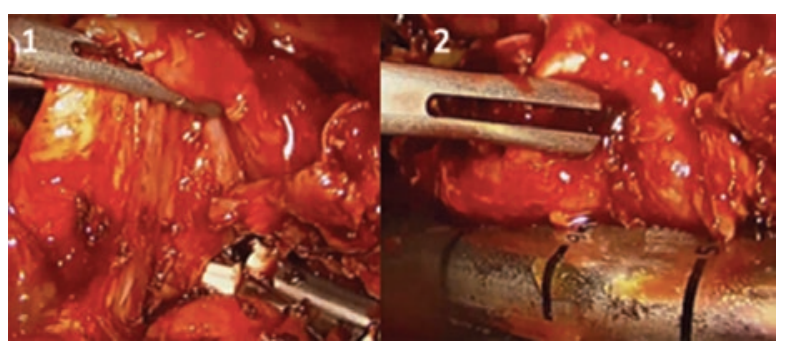

Figura 5. Duodenectomía con resección del orificio fistuloso, con preservación del páncreas y de la primera porcion duodenal y parte de la segunda.

Tabla 1. Estrategias de prevención de lesiones de vía biliar durante la colecistectomía por laparoscopia

- Visión crítica de seguridad.

- Técnica infundibular.

- Disección anterógrada.

- Colecistectomía subtotal.

- Puntos de referencia anatómicos:

- Surco de Rouvière.

- Ganglio de Calot.

- Método B-SAFE (B, bile duct; S, sulcus of Rouvière; $A$, hepatic artery; $F$, umbilical).

- Colangiografía intraoperatoria.

- Ultrasonido laparoscópico.

- Colangiografía fluorescente.

- Conversión a cirugía abierta.

primer día de posoperatorio, con drenaje biliar residual de $50 \mathrm{~cm}^{3}$ hasta su cese y posterior retiro, y una estancia hospitalaria de 5 días para cubrimiento antibiótico. No se evidencian complicaciones asociadas, como infección u obstrucción biliar, a los 12 meses de la intervención.

Nos permitimos presentar en vídeo el abordaje quirúrgico: https://youtu.be/uUMvZZ2E0GI.

\section{Discusión}

A pesar de que se han desarrollado numerosas estrategias (Tabla 1) para prevenir la lesión de la vía 
biliar durante la colecistectomía por laparoscopia, las tasas de lesión de vía biliar no han disminuido y se siguen presentando cada vez más casos debido al aumento del número de colecistectomías realizadas en todo el mundo. Las consecuencias de las lesiones de vía biliar han sido estudiadas extensivamente y son devastadoras para el paciente. La morbimortalidad aumenta sustancialmente y puede ser aún mayor si hay fuga biliar asociada, como en nuestro caso; así lo demuestran Fong, et al ${ }^{11}$ en un reciente estudio con un $7.3 \%$ en el primer año, en comparación con un $1.3 \%$ en pacientes con lesión de vía biliar sin fuga asociada.

El manejo quirúrgico de las lesiones de la vía biliar y su éxito dependen de varios factores, como la experiencia del cirujano, la identificación de la lesión en el mismo tiempo quirúrgico, la reparación temprana, la infección intraabdominal desarrollada, el nivel donde ocurra la lesión y la complejidad de esta, ya que en muchos casos se puede asociar a lesión vascular. Uno de los más importantes y determinantes factores es si la lesión es reparada por un cirujano con suficiente experiencia en reconstrucción biliar 0 por un cirujano hepatobiliar, en cuyo caso las tasas de éxito quirúrgico pueden alcanzar el $91 \%$, en comparación con el $13 \%$ en cirujanos inexpertos ${ }^{12,13}$, y lo mismo pasa con los costos, que se reducen un $29 \%$ si la reparación es realizada por un cirujano experto ${ }^{14}$. Por ello, la recomendación en general es la remisión del paciente a un centro de referencia. Otro factor determinante es el reconocimiento temprano de la lesión y si es posible su corrección quirúrgica en el mismo tiempo, pero tan solo un $25-30 \%$ de las lesiones se ven durante la cirugía, y no todos los cirujanos realizan colangiografía intraoperatoria ${ }^{15}$. La derivación tardía ( $>72$ horas después de la lesión) se asocia con una tasa significativamente más alta de abscesos intraabdominales y una mayor duración de la estadía en la unidad de cuidados intensivos, lo cual no sucedió en nuestro caso probablemente porque la fistula se encontraba conducida desde la primera intervención por un drenaje abdominal debido a su reconocimiento temprano ${ }^{16}$. A pesar de esto, varios estudios sugieren que diferir el manejo quirúrgico con la colocación de drenajes abdominales para manejar la fuga biliar, al menos 6 semanas después de la lesión, disminuye la inflamación y esto resulta en una mejoría del campo quirúrgico hostil ${ }^{17}$. En un estudio retrospectivo publicado en 2010 por Sahajpal, et al. ${ }^{18}$ se evaluó a 69 pacientes con lesiones principalmente de tipo $\mathrm{E}$ de Strasberg y se investigó el momento óptimo de reparación. Se demostró que las reparaciones tempranas, realizadas dentro de las primeras 72 horas de la lesión, o después de 6 semanas, tenían significativamente menos estenosis a largo plazo en comparación con las reparaciones intermedias (entre 72 horas y 6 semanas después de la lesión inicial), pero en este caso se encontraba asociada una fistula duodenal por el intento fallido de la derivación bilioentérica en el primer tiempo quirúrgico, y por eso se decidió la reparación antes de ese tiempo.

El manejo de los diferentes tipos de lesiones de la vía biliar es multidisciplinario. El control del foco séptico con drenaje quirúrgico o percutáneo de la colección intrabdominal es el paso inicial. El manejo definitivo puede ser endoscópico por colangiopancreatografía retrógrada endoscópica (CPRE), anterógrado percutáneo o quirúrgico, y esto depende básicamente del tipo de lesión. El manejo anterógrado percutáneo (requiere dilatación de la vía biliar intrahepática) o retrógrado endoscópico, por ejemplo, puede ser efectivo hasta en el $98 \%$ de los pacientes con lesiones Strasberg A, en los que la fuga biliar es resuelta con la colocación de un stent y la disminución de la presión con la esfinterotomía ${ }^{19}$, y los pacientes con estenosis biliares iatrogénicas con o sin fuga asociada también pueden ser manejados por vía endoscópica con dilataciones neumáticas y colocación de stents seriados de manera satisfactoria ${ }^{20}$. En un estudio con 19 pacientes con lesiones iatrogénicas de la vía biliar Strasberg C, con fuga biliar y un conducto aislado que no era posible opacificar durante la CPRE, la canulación al conducto aislado se obtuvo por vía transcística y colocación de stent, con una tasa de resolución del $100 \%$ y una recurrencia de la fuga biliar atribuible a un desplazamiento del stent metálico ${ }^{21}$.

En los demás tipos de lesiones de vía biliar, el manejo quirúrgico es el método de referencia. Existen varios tipos de reparación, incluyendo el cierre primario de la lesión, la ligadura de conductos, la anastomosis terminoterminal, la derivación bilioentérica, el cierre sobre tubo en T, hepatectomías parciales o trasplante; la elección dependerá del nivel y el tipo de la lesión ${ }^{17}$. Las transecciones de conductos menores de $3 \mathrm{~mm}$ pueden ser ligadas sin ningún riesgo de secuela hepática posterior ${ }^{22}$. Las lesiones con fuga biliar menores del $50 \%$ de la circunferencia del conducto pueden ser reparadas con cierre sobre un tubo en $\mathrm{T}$, por lo general con una sutura de monofilamento absorbible 4-023. La anastomosis terminoterminal es viable siempre y 
cuando no exista una pérdida considerable de tejido que permita realizar una anastomosis libre de tensión; la principal complicación es la estenosis posoperatoria, que se puede documentar hasta en un $10 \%$ de los pacientes, pero con la ventaja de que pueden manejarse endoscópicamente con dilatación y colocación de un stent. El método de referencia entre los diversos tipos de reparación de lesiones de la vía biliar es la reconstrucción o derivación biliodigestiva tipo hepatoyeyunostomía en $Y$ de Roux, la cual permite la reparación de lesiones más complejas en las que encontramos una pérdida importante de tejidos que no permite una reparación primaria $o$ anastomosis terminoterminal, o la localización de las lesiones por encima de la confluencia de los hepáticos incluyendo su anastomosis a los conductos hepáticos por separado. Las tasas de estenosis de la hepatoyeyunostomía varían entre un $4 \%$ y un $38 \%$, aunque la mayoría son manejadas efectivamente con dilatación transhepática y pueden requerir revisiones hasta en un $25 \%$ de los casos. En nuestra paciente, la lesión se encontraba en la confluencia de los hepáticos con extensión al conducto hepático derecho, clasificándose como una lesión de tipo E4 de la clasificación de Strasberg, y adicionalmente, por el intento de coledocoduodenostomía fallida para el manejo de la lesión inicial, se encontraba con una fístula duodenal asociada, lo cual generó un gran componente inflamatorio en los tejidos, pero además ayudó a tomar la decisión de realizar manejo quirúrgico laparoscópico a los 8 días posoperatorios, y teniendo en cuenta la experiencia del cirujano. Por lo friable del tejido duodenal, la edad de la paciente, no encontrarse en choque séptico ni en sepsis, y con buen estado nutricional, además de la reconstrucción bilioentérica con hepatoyeyunostomía, no se consideró el cierre primario duodenal ni otra técnica de manejo de lesiones duodenales diferente a la duodenectomía con preservación del páncreas y reconstrucción del tracto gastrointestinal con anastomosis gastroyeyunal en una misma asa a una distancia mayor de $60 \mathrm{~cm}$ para evitar el reflujo biliar. En un estudio de Di Saverio, et al. ${ }^{24}, 10$ pacientes con perforaciones duodenales mayores secundarias a iatrogenia, trauma o ulcera péptica en la primera o segunda porción fueron manejados con duodenectomía con preservación del páncreas sin necesidad de reconstrucción biliar, por vía laparoscópica en un paciente y en los restantes por vía abierta. El tiempo promedio de la intervención fue de 264 minutos y la estancia hospitalaria en promedio fue de 17.8 días. Se observaron complicaciones mayores en 6 pacientes, la reintervención fue

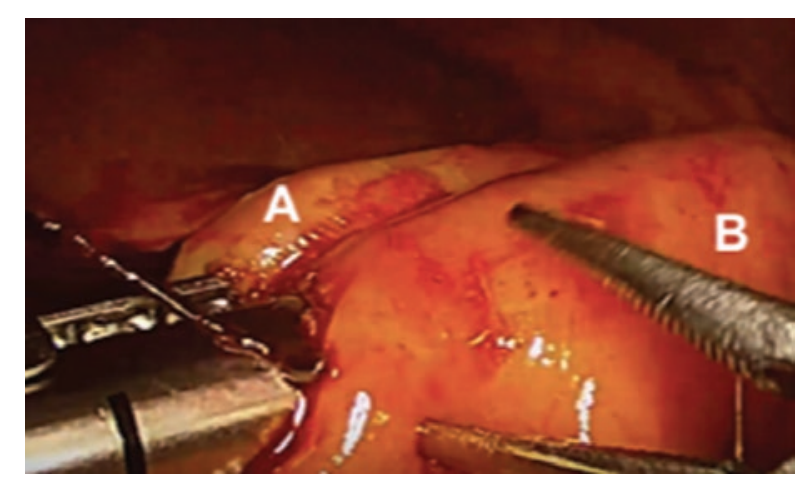

Figura 6. Anastomosis gastroyeyunal en una sola asa. A: estomago. B: yeyuno.

necesaria en 1 paciente y murieron 2 pacientes por las complicaciones. La coledocoduodenostomía como derivación bilioentérica es un procedimiento seguro cuando la indicación es correcta; tiene complicaciones frecuentes, como síndrome del sumidero, colangitis por reflujo duodenal, estenosis o fistulas en muy baja proporción. En un estudio realizado por Priego25 en el año 2013 de pacientes llevados a coledocoduodenostomía laparoscopica por patología biliar benigna, excluyendo lesiones de vía biliar reportan tasas de fuga biliar menor al $1 \%$, en este caso consideramos que muy probablemente el fallo de la coledocoduodenostomía es secundario a la altura inicial de la lesión, con lo cual, a pesar de realizar una liberación duodenal extensiva, la anastomosis no queda libre de tensión y predispone a la fuga. La coledocoduodenostomía como derivación bilioentérica en pacientes con lesión iatrogénica de la vía biliar podría ser considera en aquellos con lesiones a nivel del conducto biliar común y no en los conductos hepáticos ${ }^{12}$. Actualmente no hay reportes de casos manejados de modo similar al nuestro; la mayoría de las publicaciones hacen referencia al manejo por laparoscopia o abierto, por separado, de reconstrucciones de lesiones iatrogénicas de vía biliar o de perforaciones duodenales.

\section{Conclusión}

La lesión iatrogénica de la vía biliar es una de las mayores complicaciones quirúrgicas en la era de la colecistectomía laparoscópica. Su identificación, el control infeccioso y el manejo temprano o diferido quirúrgico, siempre y cuando se encuentre en manos de cirujanos expertos, traen consigo resultados favorables para los pacientes, sea por vía laparoscópica (como en nuestro caso) o abierta. Además, después 
del fallo de una coledocoduodenostomía, la resección duodenal con preservación del páncreas asociada a una reconstrucción biliogastrointestinal es posible y puede ser considerada en este tipo de casos.

\section{Agradecimientos}

Los autores agradecen a la paciente por su voto de confianza al permitirnos realizar un procedimiento de tan alta complejidad, y su voluntad por salir adelante.

\section{Financiamiento}

Los autores declaran no haber recibido financiación para la realización de este trabajo.

\section{Conflicto de intereses}

Los autores declaran no tener ninguna relación financiera ni personal con otras personas $u$ organizaciones que pudieran dar lugar a conflicto de intereses.

\section{Responsabilidades éticas}

Protección de personas y animales. Los autores declaran que los procedimientos seguidos se conformaron a las normas éticas del comité de experimentación humana responsable y de acuerdo con la Asociación Médica Mundial y la Declaración de Helsinki.

Confidencialidad de los datos. Los autores declaran que han seguido los protocolos de su centro de trabajo sobre la publicación de datos de pacientes.

Derecho a la privacidad y consentimiento informado. Los autores han obtenido el consentimiento informado de los pacientes y/o sujetos referidos en el artículo. Este documento obra en poder del autor de correspondencia.

\section{Bibliografía}

1. Calland JF, Tanaka K, Foley E, Bovbjerg VE, Markey DW, Blome S, et al. Outpatient laparoscopic cholecystectomy: patient outcomes after implementation of a clinical pathway. Ann Surg. 2001;233:704-15.

2. Shea JA, Berlin JA, Bachwich DR, Staroscik RN, Malet PF, McGuckin M, et al. Indications for and outcomes of cholecystectomy: a comparison of the pre and postlaparoscopic eras. Ann Surg. 1998;227:343-50.
3. Altieri MS, Brunt LM. Elimination of bile duct injury in cholecystectomy. Adv Surg. 2019;53:145-60.

4. Altieri, MS, Brunt LM, Yang, J, Zhu C, Talamini M, Pryor A. Early cholecystectomy $(<72 \mathrm{~h})$ is associated with lower rate of complications and bile duct injury: a study of 109,862 cholecystectomies in the state of New York. Surg Endosc. 2020;34(7):3051-6.

5. Giger UF, Michel JM, Opitz I, Inderbitzin D, Kocher T, Krahenbuhl L, et al. Risk factors for perioperative complications in patients undergoing laparoscopic cholecystectomy: analysis of 22,953 consecutive cases from the Swiss Association of Laparoscopic and Thoracoscopic Surgery database. J Am Coll Surg. 2006;203:723-8.

6. Strasberg SM, Hertl M, Soper NJ. An analysis of the problem of biliary injury during laparoscopic cholecystectomy. J Am Coll Surg. 1995;180:101-25.

7. Kaman L, Sanyal S, Behera A, Singh R, Katariya RN. Comparison of major bile duct injuries following laparoscopic cholecystectomy and open cholecystectomy. ANZ J Surg. 2006;76:788-91.

8. Pitt HA, Sherman S, Johnson MS. Improved outcomes of bile duct injuries in the 21st century. Ann Surg. 2013;258:490-9.

9. Booij KAC, de Reuver PR, van Dieren S, van Delden OM, Rauws EA, Busch OR, et al. Long-term impact of bile duct injury on morbidity, mortality, quality of life, and work related limitations. Ann Surg. 2018;268:143-50.

10. Yokoe M, Hata J, Takada T, Strasberg SM, Asbun HJ, Wakabayashi G, et al. Tokyo Guidelines 2018: diagnostic criteria and severity grading of acute cholecystitis (with videos). J Hepatobiliary Pancreat Sci. 2018;25(1):41-54.

11. Fong ZV, Pitt HA, Strasberg SM, Loehrer AP, Sicklick JK, Talamini MA, et al.; the California Cholecystectomy Group. Diminished survival in patients with bile leak and ductal injury: management strategy and outcomes. J Am Coll Surg. 2018;226:568e577.

12. Stewart L, Way LW. Laparoscopic bile duct injuries: timing of surgical repair does not influence success rate. A multivariate analysis of factors influencing surgical outcomes. HPB. 2009;11:516-22.

13. Felekouras E, Petrou A, Neofytou K, Moris D, Dimitrokallis N, Bramis K et al. Early or delayed intervention for bile duct injuriesfollowing laparoscopic cholecystectomy? A dilemma looking for an answer. Gastroenterol Res Pract. 2015;2015: 104235.

14. Dageforde LA, Landman MP, Feurer ID, Poulose B, Pinson CW, Moore DE. A cost-effectiveness analysis of early vs late reconstruction of iatrogenic bile duct injuries. J Am Coll Surg. 2012;214:919-27.

15. Stewart L. latrogenic biliary injuries: identification, classification, and management. Surg Clin North Am. 2014;94:297-310.

16. Fischer CP, Fahy BN, Aloia TA, Bass BL, Gaber AO, Ghobrial RM. Timing of referral impacts surgical outcomes in patients undergoing repair of bile duct injuries. HPB. 2009;11:32-7.

17. Cohen JT, Charpentier KP, Beard RE. An update on iatrogenic biliary injuries identification, classification, and management. Surg Clin N Am. 2019;99:283-99.

18. Sahajpal AK, Chow SC, Dixon E, Greig PD, Gallinger S, Wei AC. Bile duct injuries associated with laparoscopic cholecystectomy: timing of repair and long-term outcomes. Arch Surg. 2010;145:757-63.

19. Fasoulas K, Zavos C, Chatzimavroudis G, Trakateli C, Vasiliadis T, Ioannidis $A$, et al. Eleven-year experience on the endoscopic treatment of post-cholecystectomy bile leaks. Ann Gastroenterol. 2011;24:200-5.

20. Rainio M, Lindström O, Udd M, Haapamäki C, Nordin A, Kylänpää L. Endoscopic therapy of biliary injury after cholecystectomy. Dig Dis Sci. 2017;63:474-80.

21. Mutignani M, Dioscoridi L, Pugliese F, Cintolo M, Massad M, Forti E. Primary endotherapy for Strasberg type C bile leaks. Surgery; 2019;166:286-9.

22. Brunicardi FC, Anderson DK, Billiar TR, Dunn DL, Hunter JG, Matthews JB, et al. Schwartz's principles of surgery. 10th ed. New York: McGraw-Hill; 2015. p. 1332-4.

23. Cameron JL, Cameron AM. Current surgical therapy. 11th ed. Philadelphia: Elsevier; 2014. p. 383-91.

24. Di Saverio S, Segalini E, Birindelli A, Todero S, Podda M, Rizzuto A, et al. Pancreas-sparing, ampulla-preserving duodenectomy for major duodenal (D1-D2) perforations. Br J Surg. 2018;105:1487-92.

25. Priego P. Laparoscopic choledochoduodenostomy in the management of obstructive biliary tract in the ERCP era. Clin Surg. 2016;22:101-4. 